\title{
On the Smoothness of the Noncommutative Pillow and Quantum Teardrops ${ }^{\star}$
}

Tomasz BRZEZIŃSKI

Department of Mathematics, Swansea University, Singleton Park, Swansea SA2 8PP, UK

E-mail:T.Brzezinski@swansea.ac.uk

Received December 03, 2013, in final form February 09, 2014; Published online February 14, 2014

http://dx.doi.org/10.3842/SIGMA.2014.015

\begin{abstract}
Recent results by Krähmer [Israel J. Math. 189 (2012), 237-266] on smoothness of Hopf-Galois extensions and by Liu [arXiv:1304.7117] on smoothness of generalized Weyl algebras are used to prove that the coordinate algebras of the noncommutative pillow orbifold [Internat. J. Math. 2 (1991), 139-166], quantum teardrops $\mathcal{O}\left(\mathbb{W P}_{q}(1, l)\right)[$ Comm. Math. Phys. 316 (2012), 151-170], quantum lens spaces $\mathcal{O}\left(L_{q}(l ; 1, l)\right)$ [Pacific J. Math. 211 (2003), 249-263], the quantum Seifert manifold $\mathcal{O}\left(\Sigma_{q}^{3}\right)$ [J. Geom. Phys. 62 (2012), 1097-1107], quantum real weighted projective planes $\mathcal{O}\left(\mathbb{R P}_{q}^{2}(l ; \pm)\right)[$ PoS Proc. Sci. (2012), PoS(CORFU2011), 055, 10 pages] and quantum Seifert lens spaces $\mathcal{O}\left(\Sigma_{q}^{3}(l ;-)\right)[$ Axioms $\mathbf{1}$ (2012), 201-225] are homologically smooth in the sense that as their own bimodules they admit finitely generated projective resolutions of finite length.
\end{abstract}

Key words: smooth algebra; generalized Weyl algebra; strongly graded algebra; noncommutative pillow; quantum teardrop; quantum lens space; quantum real weighted projective plane

2010 Mathematics Subject Classification: 58B32; 58B34

Dedicated to Marc Rieffel on the occasion of his 75th birthday.

\section{Introduction and tools}

This note is intended to illustrate the claim that (often) a $q$-deformation of a non-smooth classical variety or an orbifold produces an algebra which has properties of the coordinate algebra of a non-commutative smooth variety or manifold. More precisely, we say that an algebra $B$ (over an algebraically closed field $\mathbb{K}$ ) is homologically smooth or simply smooth provided that as a $B$-bimodule it has a finitely generated projective resolution of finite length; see [20, Erratum]. We prove that several classes of examples of coordinate algebras of $q$-deformed orbifolds are homologically smooth. To achieve this aim we use techniques developed in [13], which are applicable to principal comodule algebras [6], and those of [14], which are applicable to generalized Weyl algebras [1]. We summarize these presently.

In [13, Corollary 6] Krähmer gives a criterion for smoothness of quantum homogeneous spaces, which through an immediate extension to a more general class of Hopf-Galois extensions and then specification to strongly group-graded algebras, provides us with a tool for showing the smoothness of the noncommutative pillow algebra studied in [2], the quantum lens space algebras $\mathcal{O}\left(L_{q}(l ; 1, l)\right)$ introduced in [12], the quantum teardrop algebras [5], the coordinate algebras of quantum real weighted projective planes $\mathcal{O}\left(\mathbb{R P}_{q}(l ;-)\right)$ defined in [3], the quantum Seifert manifold $\mathcal{O}\left(\Sigma_{q}^{3}\right)[7]$ and the quantum Seifert lens spaces $\mathcal{O}\left(\Sigma_{q}^{3}(l ;-)\right)$ [4]. More specifically, let $G$ be a group (with the neutral element $e$ ). A $G$-graded algebra $A=\oplus_{g \in G} A_{g}$ is said to be strongly

\footnotetext{
${ }^{\star}$ This paper is a contribution to the Special Issue on Noncommutative Geometry and Quantum Groups in honor of Marc A. Rieffel. The full collection is available at http://www.emis.de/journals/SIGMA/Rieffel.html
} 
graded if, for all $g, h \in G, A_{g} A_{h}=A_{g h}$. For such algebras, Krähmer's criterion for smoothness takes the following form.

Criterion 1. Let $A$ be a strongly G-graded algebra and set $B=A_{e}$. If the enveloping algebra $\mathcal{E} A:=A \otimes A^{\mathrm{op}}$ of $A$ is left Noetherian of finite global dimension, then the enveloping algebra of $B$ is also left Noetherian of finite global dimension. Consequently, B is a homologically smooth algebra.

Although [13, Corollary 6$]$ is formulated for quantum homogeneous spaces obtained via surjective homomorphism of Hopf algebras with a cosemisimple codomain, the proof extends immediately to all faithfully flat Hopf-Galois extensions $B \subseteq A$ or principal comodule algebras such that $B$ is a direct summand of $A$ as a $B$-bimodule. In the case of $G$-graded algebras, $B=A_{e}$ is a direct summand of $A$ as a $B$-bimodule, and [16, Proposition AI.3.6] ensures that a strongly $G$ graded algebra is a principal $\mathbb{K} G$-comodule algebra. In particular $A$ is projective and faithfully flat as a left and right $B$-module. The way Criterion 1 is stated indicates its iterative nature which is implicit in the proof of [13, Corollary 6]. Note in passing that the assumption about the dimension of $\mathcal{E} A$ is not necessary to conclude that $\mathcal{E} B$ is left Noetherian.

An effective way of checking whether a graded $G$-algebra $A$ is strongly graded is described in [16, Section AI.3.2]:

Lemma 1. $A=\oplus_{g \in G} A_{g}$ is strongly graded if and only if there exists a function $\omega: G \rightarrow A \otimes A$ such that

(a) for all $g \in G, \omega(g) \in A_{g^{-1}} \otimes A_{g}$,

(b) for all $g \in G, \mu \circ \omega(g)=1$, where $\mu$ is the multiplication map of $A$.

Furthermore, if $G$ is a cyclic group, then conditions $(a)$ and $(b)$ need only be checked for a generator $g$ of $G$. If $\omega(g)=\sum_{i} \omega_{i}^{\prime} \otimes \omega_{i}^{\prime \prime}$, satisfies $(a)$ and $(b)$, then $\omega$ is defined by setting $\omega(e)=1 \otimes 1$ and

$$
\omega\left(g^{n+1}\right)=\sum_{i} \omega_{i}^{\prime} \omega\left(g^{n}\right) \omega_{i}^{\prime \prime}, \quad \text { for all } \quad n>0 .
$$

A function $\omega$ satisfying conditions $(a)$ and $(b)$ in Lemma 1 is a predecessor of a strong connection form on a principal comodule algebra; see $[6,8,11]$.

Let $R$ be an algebra, let $p$ be an element of the centre of $R$ and let $\pi$ be an automorphism of $R$. The (degree-one) generalized Weyl algebra $R(\pi, p)$ is the extension of $R$ by generators $x_{+}, x_{-}$ subject to the relations, for all $r \in R$,

$$
x_{-} x_{+}=p, \quad x_{+} x_{-}=\pi(p), \quad x_{ \pm} r=\pi^{ \pm 1}(r) x_{ \pm} ;
$$

see [1]. In [14, Theorem 4.5] Liu gives the following criterion of smoothness of a generalized Weyl algebra over the polynomial algebra.

Criterion 2. Let $R=\mathbb{K}[a]$ be a polynomial algebra and an automorphism $\pi: \mathbb{K}[a] \rightarrow \mathbb{K}[a]$ be determined by $\pi(a)=\kappa a+\chi$. Then the generalized Weyl algebra $R(\pi, p)$ is homologically smooth with homological dimension 2 if and only if the polynomial $p \in \mathbb{K}[a]$ has no multiple roots.

Furthermore, Liu proves that if the smoothness Criterion 2 is satisfied, then $A=R(\pi, p)$ is a twisted Calabi-Yau algebra of dimension 2 with the Nakayama (twisting) automorphism $\nu: A \rightarrow A$ given by $\nu\left(x_{ \pm}\right)=\kappa^{ \pm 1} x_{ \pm}$and $\nu(a)=a$. This means that the Hochschild cohomology of $A$ with values in its enveloping algebra is trivial in all degrees except degree 2 , where it is equal to $A$ with the $A$-bimodule structure $a \cdot b \cdot a^{\prime}=a b \nu\left(a^{\prime}\right)$.

The reader should observe that, except for some special cases, the algebras described hereinafter are not smooth whenever the deformation parameters $\lambda$ or $q$ are equal to 1 . By noting this they will fully grasp the main message of this note, namely that deformation may (and quite often does) result in smoothing classically singular objects. 


\section{Results}

Throughout we work with associative complex $*$-algebras with identity. We write $\mathcal{E} A$ for the enveloping algebra $A \otimes A^{\text {op }}$ of $A$. We often use the $q$-Pochhammer symbol which, for an indeterminate $x$ and a complex number $q$, is defined as

$$
(x ; q)_{n}:=\prod_{m=0}^{n-1}\left(1-q^{m} x\right) .
$$

\subsection{The noncommutative pillow}

Let $\lambda=e^{2 \pi i \theta}$, where $\theta$ is an irrational number. Recall that the coordinate $*$-algebra $\mathcal{O}\left(\mathbb{T}_{\theta}^{2}\right)$ of the noncommutative torus is generated by unitaries $U, V$, such that $U V=\lambda V U$; see [18]. The involutive algebra automorphism given by

$$
\sigma: \mathcal{O}\left(\mathbb{T}_{\theta}^{2}\right) \rightarrow \mathcal{O}\left(\mathbb{T}_{\theta}^{2}\right), \quad U \mapsto U^{*}, \quad V \mapsto V^{*}
$$

makes $\mathcal{O}\left(\mathbb{T}_{\theta}^{2}\right)$ into a $\mathbb{Z}_{2}$-graded algebra. The fixed point (or degree-zero) subalgebra $\mathcal{O}\left(P_{\theta}\right)$ is generated by $U+U^{*}$ and $V+V^{*}$. It has been introduced and studied from a topological point of view in [2] (see also [9, Section 3.7]) as a deformation of the coordinate algebra of the pillow orbifold [19, Chapter 13] (an orbifold rather than manifold since, classically, the $\mathbb{Z}_{2}$-action determined by the automorphism $\sigma$ is not free).

Theorem 1. $\mathcal{O}\left(\mathbb{T}_{\theta}^{2}\right)$ is a strongly $\mathbb{Z}_{2}$-graded algebra and the noncommutative pillow algebra $\mathcal{O}\left(P_{\theta}\right)$ is homologically smooth.

Proof. Set

$$
\hat{x}=U-U^{*}, \quad \hat{y}=V-V^{*}, \quad \hat{z}=U V^{*}-U^{*} V .
$$

Note that $\sigma(\hat{x})=-\hat{x}, \sigma(\hat{y})=-\hat{y}$ and $\sigma(\hat{z})=-\hat{z}$, i.e. all these are homogeneous elements of $\mathcal{O}\left(\mathbb{T}_{\theta}^{2}\right)$ with the $\mathbb{Z}_{2}$-degree 1 . A straightforward calculation affirms that these elements satisfy the following relation

$$
\hat{x}^{2}+\hat{y}^{2}-\bar{\lambda} \hat{z}^{2}-\hat{x} z \hat{y}=2\left(\bar{\lambda}^{2}-1\right),
$$

where $z=U V^{*}+U^{*} V \in \mathcal{O}\left(P_{\theta}\right)$. Therefore, the mapping $\omega: \mathbb{Z}_{2} \rightarrow \mathcal{O}\left(\mathbb{T}_{\theta}^{2}\right) \otimes \mathcal{O}\left(\mathbb{T}_{\theta}^{2}\right)$, defined as $\omega(0)=1 \otimes 1$ and

$$
\omega(1)=\frac{1}{2\left(\bar{\lambda}^{2}-1\right)}(\hat{x} \otimes \hat{x}+\hat{y} \otimes \hat{y}-\bar{\lambda} \hat{z} \otimes \hat{z}-\hat{x} z \otimes \hat{y})
$$

satisfies conditions $(a)$ and $(b)$ in Lemma 1 , and $\mathcal{O}\left(\mathbb{T}_{\theta}^{2}\right)$ is a strongly $\mathbb{Z}_{2}$-graded algebra.

Both $\mathcal{O}\left(\mathbb{T}_{\theta}^{2}\right)$ and $\mathcal{E O}\left(\mathbb{T}_{\theta}^{2}\right)$ can be understood as iterated skew Laurent polynomial rings and hence they are left Noetherian by [15, Theorem 1.4.5]. Furthermore, the global dimension of the latter is less than or equal to 4 by [15, Theorem 7.5.3]. Therefore, the noncommutative pillow algebra $\mathcal{O}\left(P_{\theta}\right)$ is homologically smooth by Criterion 1 .

In short, Theorem 1 means that for the irrational $\theta$ (or, more generally, for any real $\theta \in$ $\left.(0,1) \backslash\left\{\frac{1}{2}\right\}\right)$ the action of $\mathbb{Z}_{2}$ on the noncommutative torus is free despite the fact that the corresponding action on the classical level is not free. The set of fixed points corresponds to a manifold rather than an orbifold. 


\subsection{Quantum teardrops and lens spaces}

Here we deal with three (classes of) complex $*$-algebras given in terms of generators and relations.

The coordinate algebra of the quantum three-sphere, $\mathcal{O}\left(S_{q}^{3}\right)$, is generated by $\alpha$ and $\beta$ such that

$$
\begin{array}{ll}
\alpha \beta=q \beta \alpha, \quad \alpha \beta^{*}=q \beta^{*} \alpha, & \beta \beta^{*}=\beta^{*} \beta, \\
\alpha \alpha^{*}=\alpha^{*} \alpha+\left(q^{-2}-1\right) \beta \beta^{*}, & \alpha \alpha^{*}+\beta \beta^{*}=1,
\end{array}
$$

where $q \in(0,1)$; see [21]. For any positive integer $l$, the coordinate algebra of the quantum lens space $\mathcal{O}\left(L_{q}(l ; 1, l)\right)$ is a $*$-algebra generated by $c$ and $d$ subject to the following relations:

$$
c d=q^{l} d c, \quad c d^{*}=q^{l} d^{*} c, \quad d d^{*}=d^{*} d, \quad c c^{*}=\left(d d^{*} ; q^{2}\right)_{l}, \quad c^{*} c=\left(q^{-2} d d^{*} ; q^{-2}\right)_{l},
$$

see [12]. Finally, for a positive integer $l$, the coordinate algebra of the quantum teardrop $\mathcal{O}\left(\mathbb{W P}_{q}(1, l)\right)$ is the $*$-algebra generated by $a$ and $b$ subject to the following relations

$$
a^{*}=a, \quad a b=q^{-2 l} b a, \quad b b^{*}=q^{2 l} a\left(a ; q^{2}\right)_{l}, \quad b^{*} b=a\left(q^{-2} a ; q^{-2}\right)_{l} ;
$$

see [5]. These algebras form a tower $\mathcal{O}\left(\mathbb{W P}_{q}(1, l)\right) \hookrightarrow \mathcal{O}\left(L_{q}(l ; 1, l)\right) \hookrightarrow \mathcal{O}\left(S_{q}^{3}\right)$ with embeddings $a \mapsto d d^{*}, b \mapsto c d$ and $c \mapsto \alpha^{l}, d \mapsto \beta$, respectively. We thus can and will think of $\mathcal{O}\left(\mathbb{W P}_{q}(1, l)\right)$ and $\mathcal{O}\left(L_{q}(l ; 1, l)\right)$ as subalgebras of $\mathcal{O}\left(S_{q}^{3}\right) . \mathcal{O}\left(S_{q}^{3}\right)$ is a $\mathbb{Z}_{l}$-graded algebra with grading given by $\operatorname{deg}(\alpha)=1, \operatorname{deg}\left(\alpha^{*}\right)=l-1, \operatorname{deg}(\beta)=\operatorname{deg}\left(\beta^{*}\right)=0$, and the above embedding identifies the degree-zero part of $\mathcal{O}\left(S_{q}^{3}\right)$ with $\mathcal{O}\left(L_{q}(l ; 1, l)\right)$. By [5, Theorem 3.3], the latter is a strongly $\mathbb{Z}$-graded algebra with grading provided by $\operatorname{deg}(c)=\operatorname{deg}\left(d^{*}\right)=1, \operatorname{deg}\left(c^{*}\right)=\operatorname{deg}(d)=-1$ and with the degree-zero part isomorphic to $\mathcal{O}\left(\mathbb{W P}_{q}(1, l)\right)$.

That $\mathcal{O}\left(\mathbb{W P}_{q}(1, l)\right)$ is homologically smooth can be argued as follows. $\mathcal{O}\left(S_{q}^{3}\right)$ is a coordinate algebra of the quantum group $\mathrm{SU}(2)$ and thus $\mathcal{E O}\left(S_{q}^{3}\right)$ is left Noetherian and has a finite global dimension; see [10]. Hence, if it were a strongly $\mathbb{Z}_{l}$-graded algebra, then $\mathcal{E} \mathcal{O}\left(L_{q}(l ; 1, l)\right)$ would be left Noetherian and would have a finite global dimension (so, in particular $\mathcal{O}\left(L_{q}(l ; 1, l)\right.$ ) would be homologically smooth) by Criterion 1 . Since, in turn $\mathcal{O}\left(L_{q}(l ; 1, l)\right)$ is a strongly graded algebra, Criterion 1 would imply smoothness of the teardrop algebra $\mathcal{O}\left(\mathbb{W P}_{q}(1, l)\right)$. This arguing leads to:

Theorem 2. $\mathcal{O}\left(S_{q}^{3}\right)$ is a strongly $\mathbb{Z}_{l}$-graded algebra with the degree-zero subalgebra isomorphic to $\mathcal{O}\left(L_{q}(l ; 1, l)\right)$. Consequently, both $\mathcal{O}\left(L_{q}(l ; 1, l)\right)$ and $\mathcal{O}\left(\mathbb{W P}_{q}(1, l)\right)$ are homologically smooth algebras.

Proof. The case $l=1$ is dealt with in [13], the remaining cases follow from

Lemma 2. For all integers $l>1$, there exist elements $\omega(1) \in \mathcal{O}\left(S_{q}^{3}\right)_{l-1} \otimes \mathcal{O}\left(S_{q}^{3}\right)_{1}$ such that $\mu(\omega(1))=1$.

Proof. Set:

$$
\omega(1)=x_{1} \alpha^{l-1} \otimes \alpha^{* l-1}+\sum_{p=1}^{l-1} y_{p} a^{p-1} \alpha^{*} \otimes \alpha,
$$

where $x_{1}, y_{1}, \ldots, y_{l-1} \in \mathbb{C}$ are to be determined and $a=\beta \beta^{*}=d d^{*}$. Then $\omega(1) \in \mathcal{O}\left(S_{q}^{3}\right)_{l-1} \otimes$ $\mathcal{O}\left(S_{q}^{3}\right)_{1}$. Using (1) one finds that

$$
\alpha^{m} \alpha^{* m}=\left(a ; q^{2}\right)_{m}=: \sum_{p=0}^{m} c_{p}^{m} a^{p},
$$


where $c_{p}^{m}$ are the appropriate $q$-binomial coefficients (defined by the second equality in (2)). In view of $(1)$, the condition $\mu(\omega(1))=1$ leads to

$$
x_{1} \sum_{p=0}^{l-1} c_{p}^{l-1} a^{p}+\sum_{p=0}^{l-2} y_{p+1} a^{p}-q^{-2} \sum_{p=1}^{l-1} y_{p} a^{p}=1 .
$$

By comparing the powers of $a$, this is converted into an inhomogeneous system of $l$ equations with unknown $x_{1}, y_{1}, \ldots, y_{l-1}$, whose determinant is

$$
\Delta_{l: 1}=\left|\begin{array}{ccccccc}
c_{0}^{l-1} & 1 & 0 & 0 & \ldots & 0 & 0 \\
c_{1}^{l-1} & -q^{-2} & 1 & 0 & \ldots & 0 & 0 \\
c_{2}^{l-1} & 0 & -q^{-2} & 1 & \ldots & 0 & 0 \\
\vdots & & & \vdots & & & \\
c_{l-2}^{l-1} & 0 & 0 & 0 & \cdots & -q^{-2} & 1 \\
c_{2}^{l-1} & 0 & 0 & 0 & \ldots & 0 & -q^{-2}
\end{array}\right| .
$$

$\Delta_{l: 1}$ can be evaluated by expanding by the first column to give

$$
\Delta_{l: 1}=(-1)^{l-1}\left(q^{-2(l-1)} c_{0}^{l-1}+q^{-2(l-2)} c_{1}^{l-1}+\cdots+c_{l-1}^{l-1}\right)=\left(-q^{2}\right)^{l-1} \prod_{p=1}^{l-1}\left(1-q^{2 p}\right) \neq 0 .
$$

The final equality follows from the definition of the $q$-binomial coefficients (2). This proves the existence of $\omega(1)$ as stated.

Since 1 is a generator of $\mathbb{Z}_{l}$, Lemma 2 ensures the existence of mappings $\omega: \mathbb{Z}_{l} \rightarrow \mathcal{O}\left(S_{q}^{3}\right) \otimes$ $\mathcal{O}\left(S_{q}^{3}\right)$ that satisfy conditions $(a)$ and $(b)$ in Lemma 1 . Hence $\mathcal{O}\left(S_{q}^{3}\right)$ is a strongly $\mathbb{Z}_{l \text {-graded }}$ algebra, and the second assertion of the theorem follows by Criterion 1.

Therefore, for any $q \in(0,1)$ the action of $\mathbb{Z}_{l}$ on the quantum three-sphere described above is free despite the fact that the corresponding action on the classical level is not free (unless, obviously, $l=1)$. The fixed points correspond to a manifold rather than an orbifold.

\subsection{Odd weighted real projective planes $\mathbb{R P}_{q}^{2}(l ;-)$ and quantum Seifert lens spaces}

For a positive integer $l$, the coordinate $*$-algebra $\mathcal{O}\left(\mathbb{R P}_{q}^{2}(l ;-)\right)$ of the odd quantum weighted real projective plane is generated by $a, b, c_{-}$which satisfy the relations:

$$
\begin{aligned}
& a=a^{*}, \quad a b=q^{-2 l} b a, \quad a c_{-}=q^{-4 l} c_{-} a, \quad b^{2}=q^{3 l} a c_{-}, \quad b c_{-}=q^{-2 l} c_{-} b, \\
& b b^{*}=q^{2 l} a\left(a ; q^{2}\right)_{l}, \quad b^{*} b=a\left(q^{-2} a ; q^{-2}\right)_{l}, \quad b^{*} c_{-}=q^{-l}\left(q^{-2} a ; q^{-2}\right)_{l} b, \\
& c_{-} b^{*}=q^{l} b\left(a ; q^{2}\right)_{l}, \quad c_{-} c_{-}^{*}=\left(a ; q^{2}\right)_{2 l}, \quad c_{-}^{*} c_{-}=\left(q^{-2} a ; q^{-2}\right)_{2 l},
\end{aligned}
$$

see [3]. To prove homological smoothness of $\mathcal{O}\left(\mathbb{R P}_{q}^{2}(l ;-)\right)$ we make use of Criterion 1 and build a tower of strongly graded algebras with $\mathcal{O}\left(\mathbb{R P}_{q}^{2}(l ;-)\right)$ as the foundation.

The coordinate $*$-algebra of the quantum Seifert manifold $\mathcal{O}\left(\Sigma_{q}^{3}\right)$ is generated by a central unitary $\xi$ and elements $\zeta_{0}, \zeta_{1}$ such that

$$
\zeta_{0} \zeta_{1}=q \zeta_{1} \zeta_{0}, \quad \zeta_{0} \zeta_{0}^{*}=\zeta_{0}^{*} \zeta_{0}+\left(q^{-2}-1\right) \zeta_{1}^{2} \xi, \quad \zeta_{0} \zeta_{0}^{*}+\zeta_{1}^{2} \xi=1, \quad \zeta_{1}^{*}=\zeta_{1} \xi
$$


It has been shown in [7, proof of Proposition 5.2] that $\mathcal{O}\left(\Sigma_{q}^{3}\right)$ can be understood as the degreezero part of a $\mathbb{Z}_{2}$-grading of $\mathcal{O}\left(S_{q}^{2}\right)\left[u, u^{-1}\right]$, where $u^{-1}=u^{*}$ and $\mathcal{O}\left(S_{q}^{2}\right)$ is the coordinate $*$-algebra of the equatorial Podleś sphere [17], generated by $z_{0}$ and self-adjoint $z_{1}$ such that

$$
z_{0} z_{1}=q z_{1} z_{0}, \quad z_{0} z_{0}^{*}=z_{0}^{*} z_{0}+\left(q^{-2}-1\right) z_{1}^{2}, \quad z_{0} z_{0}^{*}+z_{1}^{2}=1 .
$$

The $\mathbb{Z}_{2}$-grading of $\mathcal{O}\left(S_{q}^{2}\right)\left[u, u^{-1}\right]$ is determined by setting, for all monomials $w$ of degree $k$ in the basis $\left\{z_{0}^{r} z_{1}^{s}, z_{0}^{* r} z_{1}^{s} \mid r, s \in \mathbb{N}\right\}$ of $\mathcal{O}\left(S_{q}^{2}\right), \operatorname{deg}\left(w u^{m}\right)=(k+m) \bmod 2 . \mathcal{O}\left(\Sigma_{q}^{3}\right)$ can be identified with the degree-zero part of $\mathcal{O}\left(S_{q}^{2}\right)\left[u, u^{-1}\right]$ by $*$-embedding $\zeta_{i} \mapsto z_{i} u, \xi \mapsto u^{-2}$. Thanks to the last of equations (4), the function

$$
\omega: \mathbb{Z}_{2} \rightarrow \mathcal{O}\left(S_{q}^{2}\right)\left[u, u^{-1}\right] \otimes \mathcal{O}\left(S_{q}^{2}\right)\left[u, u^{-1}\right], \quad 0 \mapsto 1 \otimes 1, \quad 1 \mapsto z_{0} \otimes z_{0}^{*}+z_{1} \otimes z_{1},
$$

satisfies conditions (a) and (b) in Lemma 1 , hence $\mathcal{O}\left(S_{q}^{2}\right)\left[u, u^{-1}\right]$ is a strongly $\mathbb{Z}_{2}$-graded algebra. Since $\mathcal{E} \mathcal{O}\left(S_{q}^{3}\right)$ is Noetherian, and there is a surjective $*$-algebra homomorphism $\mathcal{O}\left(S_{q}^{3}\right) \rightarrow \mathcal{O}\left(S_{q}^{2}\right)$, $\alpha \mapsto z_{0}, \beta \mapsto z_{1}^{*}$, both $\mathcal{E} \mathcal{O}\left(S_{q}^{2}\right)$ and $\mathcal{E} \mathcal{O}\left(S_{q}^{2}\right)\left[u, u^{-1}\right]$ and hence also $\mathcal{E} \mathcal{O}\left(\Sigma_{q}^{3}\right)$ are Noetherian.

As explained in [4], $\mathcal{O}\left(\Sigma_{q}^{3}\right)$ is a $\mathbb{Z}_{l}$-graded algebra with grading given by

$$
\operatorname{deg}\left(\zeta_{0}\right)=1, \quad \operatorname{deg}\left(\zeta_{0}^{*}\right)=l-1, \quad \operatorname{deg}\left(\zeta_{1}\right)=\operatorname{deg}(\xi)=0 .
$$

The degree-zero part of $\mathcal{O}\left(\Sigma_{q}^{3}\right)$ is isomorphic to the $*$-algebra $\mathcal{O}\left(\Sigma_{q}^{3}(l ;-)\right)$ generated by $x, y$ and central unitary $z$ subject to the following relations

$$
y^{*}=y z, \quad x y=q^{l} y x, \quad x x^{*}=\left(y^{2} z ; q^{2}\right)_{l}, \quad x^{*} x=\left(q^{-2} y^{2} z ; q^{-2}\right)_{l} .
$$

The embedding of $\mathcal{O}\left(\Sigma_{q}^{3}(l ;-)\right)$ into $\mathcal{O}\left(\Sigma_{q}^{3}\right)$ is given by $x \mapsto \zeta_{0}^{l}, y \mapsto \zeta_{1}$ and $z \mapsto \xi$. The similarity of relations (3) and (1) leads immediately to equations (2) with $\alpha$ replaced by $\zeta_{0}$ and $a=\zeta_{1}^{2} \xi$. This allows one to use the same arguments as in Lemma 2 to prove that there exist $x_{1}, y_{1}, \ldots, y_{l-1} \in \mathbb{C}$ such that

$$
\omega(1)=x_{1} \zeta_{0}^{l-1} \otimes \zeta_{0}^{* l-1}+\sum_{i=1}^{l-1} y_{i} a^{i-1} \zeta_{0}^{*} \otimes \zeta_{0} \in \mathcal{O}\left(\Sigma_{q}^{3}\right)_{l-1} \otimes \mathcal{O}\left(\Sigma_{q}^{3}\right)_{l}
$$

has the required property $\mu(\omega(1))=1$. Therefore, $\mathcal{O}\left(\Sigma_{q}^{3}\right)$ is a strongly graded $\mathbb{Z}_{l}$-algebra.

Finally, it is proven in [4] that $\mathcal{O}\left(\Sigma_{q}^{3}(l ;-)\right)$ is a strongly $\mathbb{Z}$-graded algebra with grading given by $\operatorname{deg}(x)=\operatorname{deg}(y)=1, \operatorname{deg}\left(x^{*}\right)=-1$ and $\operatorname{deg}(z)=-2$. The degree-zero subalgebra of $\mathcal{O}\left(\Sigma_{q}^{3}(l ;-)\right)$ can be identified with the coordinate algebra of weighted real projective plane $\mathcal{O}\left(\mathbb{R P}_{q}^{2}(l ;-)\right)$ via the map $a \mapsto y^{2} z, b \mapsto x y z$ and $c_{-} \mapsto x^{2} z$.

Summarizing, we have presented in this section a tower of $*$-algebras

$$
\mathcal{O}\left(\mathbb{R P}_{q}^{2}(l ;-)\right) \hookrightarrow \mathcal{O}\left(\Sigma_{q}^{3}(l ;-)\right) \hookrightarrow \mathcal{O}\left(\Sigma_{q}^{3}\right) \hookrightarrow \mathcal{O}\left(S_{q}^{2}\right)\left[u, u^{-1}\right]
$$

The second, third and fourth terms are strongly group graded algebras. Each antecedent term is the degree-zero part of the subsequent one. Since the enveloping algebra of $\mathcal{O}\left(S_{q}^{2}\right)\left[u, u^{-1}\right]$ is Noetherian, so are the enveloping algebras of all its predecessors. By [14, Corollary 4.6] the global dimension of $\mathcal{E} \mathcal{O}\left(S_{q}^{2}\right)$ is finite, hence so is the global dimension of $\mathcal{E} \mathcal{O}\left(S_{q}^{2}\right)\left[u, u^{-1}\right]$, and, by Criterion 1, the global dimensions of enveloping algebras of all its predecessors in (5). This proves the following

Theorem 3. The algebras $\mathcal{O}\left(\Sigma_{q}^{3}\right), \mathcal{O}\left(\mathbb{R P}_{q}^{2}(l ;-)\right)$ and $\mathcal{O}\left(\Sigma_{q}^{3}(l ;-)\right)$ are homologically smooth. 


\subsection{Quantum real weighted projective planes $\mathbb{R P}_{q}^{2}(l ;+)$ and teardrops (revisited)}

Let $k$ be a natural number and $l$ be a positive integer. Write $\mathcal{A}(k, l)$ for the $*$-algebra generated by $a$ and $b$ subject to the following relations

$$
a^{*}=a, \quad a b=q^{-2 k l} b a, \quad b b^{*}=q^{2 k l} a^{k}\left(a ; q^{2}\right)_{l}, \quad b^{*} b=a^{k}\left(q^{-2} a ; q^{-2}\right)_{l} .
$$

If $k$ and $l$ are coprime then $\mathcal{A}(k, l)$ is the coordinate algebra of the quantum weighted projective line or the quantum spindle $\mathcal{O}\left(\mathbb{W P}_{q}(k, l)\right)$ introduced in [5]. The special case $k=1$ is simply the quantum teardrop; see Section 2.2. For $l$ odd, $\mathcal{A}(0, l)$ is the coordinate algebra of the quantum weighted even real projective plane $\mathcal{O}\left(\mathbb{R P}_{q}^{2}(l ;+)\right)$ introduced in [3]. The following theorem is a consequence of Criterion 2.

Theorem 4. The algebras $\mathcal{A}(k, l)$ are homologically smooth (of dimension 2) if and only if $k=0,1$.

Proof. We only need to observe that each $\mathcal{A}(k, l)$ is a generalized Weyl algebra over the polynomial algebra $\mathbb{C}[a]$ given by the automorphism $\pi(a)=q^{2 l} a$, element $p=a^{k} \prod_{m=1}^{l}\left(1-q^{-2 m} a\right)$ and generators $x_{-}=b, x_{+}=b^{*}$. Since $p$ has no multiple roots if and only if $k=0,1$, the assertion follows by Criterion 2 .

Furthermore, for $k=0,1, \mathcal{A}(k, l)$ are twisted Calabi-Yau algebras with the twisting automorphism $\nu(b)=q^{-2 l} b, \nu\left(b^{*}\right)=q^{2 l} b^{*}$ and $\nu(a)=a$. Hence they enjoy the Poincaré duality in the sense of Van den Bergh [20].

\section{Acknowledgements}

I would like to thank Ulrich Krähmer for discussions, Li-Yu Liu for bringing reference [14] to my attention, and Piotr M. Hajac and the referees for helpful comments. I am grateful to Fields Institute for Research in Mathematical Sciences in Toronto, where these results were first presented, for creating excellent research environment and for support.

\section{References}

[1] Bavula V., Tensor homological minimal algebras, global dimension of the tensor product of algebras and of generalized Weyl algebras, Bull. Sci. Math. 120 (1996), 293-335.

[2] Bratteli O., Elliott G.A., Evans D.E., Kishimoto A., Noncommutative spheres. I, Internat. J. Math. 2 (1991), 139-166.

[3] Brzeziński T., Circle actions on a quantum Seifert manifold, PoS Proc. Sci. (2012), PoS(CORFU2011), 055, 10 pages, arXiv:1203.6801.

[4] Brzeziński T., Fairfax S.A., Bundles over quantum real weighted projective spaces, Axioms 1 (2012), 201225, arXiv:1207.2313.

[5] Brzeziński T., Fairfax S.A., Quantum teardrops, Comm. Math. Phys. 316 (2012), 151-170, arXiv:1107.1417.

[6] Brzeziński T., Hajac P.M., The Chern-Galois character, C. R. Math. Acad. Sci. Paris 338 (2004), 113-116, math.KT/0306436.

[7] Brzeziński T., Zieliński B., Quantum principal bundles over quantum real projective spaces, J. Geom. Phys. 62 (2012), 1097-1107, arXiv:1105.5897.

[8] Dąbrowski L., Grosse H., Hajac P.M., Strong connections and Chern-Connes pairing in the Hopf-Galois theory, Comm. Math. Phys. 220 (2001), 301-331, math.QA/9912239.

[9] Evans D.E., Kawahigashi Y., Quantum symmetries on operator algebras, Oxford Mathematical Monographs, Oxford Science Publications, The Clarendon Press, Oxford University Press, New York, 1998. 
[10] Goodearl K.R., Zhang J.J., Homological properties of quantized coordinate rings of semisimple groups, Proc. Lond. Math. Soc. (3) 94 (2007), 647-671, math.QA/0510420.

[11] Hajac P.M., Strong connections on quantum principal bundles, Comm. Math. Phys. 182 (1996), 579-617, hep-th/9406129.

[12] Hong J.H., Szymański W., Quantum lens spaces and graph algebras, Pacific J. Math. 211 (2003), $249-263$.

[13] Krähmer U., On the Hochschild (co)homology of quantum homogeneous spaces, Israel J. Math. 189 (2012), 237-266, arXiv:0806.0267.

[14] Liu L.-Y., Homological smoothness and deformations of generalized Weyl algebras, arXiv:1304.7117.

[15] McConnell J.C., Robson J.C., Noncommutative Noetherian rings, Graduate Studies in Mathematics, Vol. 30, American Mathematical Society, Providence, RI, 2001.

[16] Năstăsescu C., van Oystaeyen F., Graded ring theory, North-Holland Mathematical Library, Vol. 28, NorthHolland Publishing Co., Amsterdam, 1982.

[17] Podleś P., Quantum spheres, Lett. Math. Phys. 14 (1987), 193-202.

[18] Rieffel M.A., $C^{*}$-algebras associated with irrational rotations, Pacific J. Math. 93 (1981), 415-429.

[19] Thurston W.P., The geometry and topology of three-manifolds, Princeton University, 1980, available at http://www.msri.org/publications/books/gt3m/.

[20] van den Bergh M., A relation between Hochschild homology and cohomology for Gorenstein rings, Proc. Amer. Math. Soc. 126 (1998), 1345-1348, Erratum, Proc. Amer. Math. Soc. 130 (2002), 2809-2810.

[21] Woronowicz S.L., Compact matrix pseudogroups, Comm. Math. Phys. 111 (1987), 613-665. 